\title{
ORBITALS IN GENERAL CHEMISTRY, PART II: MATHEMATICAL REALITIES
}

\author{
Guy Lamoureux ${ }^{\mathrm{a}, \mathrm{b}, *,(1)}$ and John F. Ogilvie ${ }^{\mathrm{a}, \mathrm{c}, \mathrm{d}}$ \\ aUniversidad de Costa Rica, Escuela de Química, 11501-2060 San Pedro de Montes de Oca, San José, Costa Rica

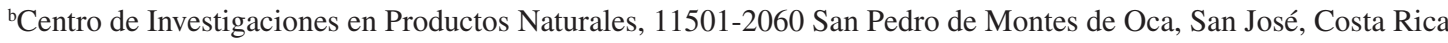 \\ ${ }^{\mathrm{c} C e n t r e}$ for Experimental and Constructive Mathematics, Department of Mathematics, Simon Fraser University, 8888 University \\ Drive, Burnaby, British Columbia V5A 1S6 Canada \\ Institute of Quantum Physics, Irkutsk National Research Technical University, 83 Lermontov Street, Irkutsk 664074, Russian \\ Federation
}

Recebido em 14/06/2020; aceito em 29/09/2020; publicado na web em 04/11/2020

\begin{abstract}
In Part II of a three-part series, we discuss two factors absent from textbooks of general chemistry that are important in a discussion of teaching orbitals. First, atomic orbitals are shown systematically to comprise algebraic formulae in coordinates of not one but four sets (spherical polar, paraboloidal, ellipsoidal, spheroconical coordinates). Each formula has its corresponding shape as a surface of constant amplitude; some visual examples are provided. Second, the argument that molecular structure is incompatible with quantum mechanics is presented. Despite the utility of orbitals as mathematical functions in various calculations, they are intrinsically complicated for the traditional purpose of qualitative explanation of molecular structure.
\end{abstract}

Keywords: teaching general chemistry; orbitals; quantum mechanics; molecular structure.

\section{INTRODUCTION}

Science is concerned only with observable things. - P. A. M. Dirac ${ }^{1}$

From Part I we recall a definition of an orbital according to a reputable textbook ${ }^{2}$ for general chemistry: "the solutions to Schroedinger's equation for the hydrogen atom yield a set of wave functions called orbitals". That definition is generally acceptable and approximately correct: the solutions number four sets ${ }^{3}$ rather than one. Moreover, according to Schroedinger, ${ }^{4}$ these functions in any set independent of time are named amplitude functions, not wave functions, to which usage we accordingly conform. Here in Part II we present explicitly these orbitals as algebraic formulae in four sets as an unequivocal proof of their reality as equivalent mathematical entities, and discuss the relevance of this multiplicity in the light of quantum-mechanical principles. A general expression for the orbitals according to the spatial coordinates of four systems in which they have been derived is accompanied in each case with an explicit single instance of an actual algebraic formula and its depiction as a surface of constant amplitude. We make no apology for presenting here extensive algebraic formulae - orbitals are neither more than, nor less than, mathematical functions, so not observable properties; each chemist must become reconciled to this fact of science.

We show below that most confusion about orbitals is based on an enduring misconception that quantum mechanics provides a mathematically unique representation. In systems of two or more electrons, the orbital configurations cannot be considered to be unique ${ }^{5}$ the same condition is true of atomic orbitals derived for a one-electron system, as we show here. In 1983 McKelvey asserted prophetically that some organic chemists would be upset to learn that aspects of Dirac's relativistic quantum mechanics - quantum numbers, representation of orbitals, absence of nodes - fail to exhibit a correlation one to one with non-relativistic quantum mechanics. ${ }^{6}$
How do chemists feel when they realize that, even according to Schroedinger's wave mechanics in coordinate space, there are four variations on a theme of orbitals, as we proceed to demonstrate? More importantly, what is the consequence of teaching this increasingly complicated subject to first-year students?

\section{DISCUSSION}

\section{Systems of coordinates and exemplary orbitals}

In coordinates as spatial variables in exactly four systems, ${ }^{3}$ Schroedinger's partial-differential equation independent of time for the hydrogen atom becomes separable into three ordinary-differential equations that have unique solutions in well-defined and explicit standard algebraic formulae, as follows.

\section{Spherical polar coordinates}

The coordinates in this system, ${ }^{7}$ the only system known to almost all chemists, are conventionally named $r, \theta, \phi$; a surface of constant $r$ corresponds in Cartesian coordinates to a finite sphere of that radius with its center at the origin of the coordinate system; a surface of constant $\theta$ corresponds to an infinite cone of circular cross section about axis $z$ with angle $\theta$ from that axis and its vertex at the origin; a surface of constant $\phi$ corresponds to an infinite half-plane from axis $z$. These features are illustrated in Figure 1. Please be aware that, although one can directly undertake mathematical operations in various systems of coordinates, all pictorial representations must appear in Cartesian coordinates with which human eyes are familiar; advanced mathematical software (Maple for our work) readily fulfills these requirements. On this basis all our plots of orbitals are directly comparable.

The solutions $\psi_{k, l, m}(r, \theta, \phi)$ to the Schroedinger equation independent of time in these spherical polar coordinates, i.e. the orbitals, are expressible as 


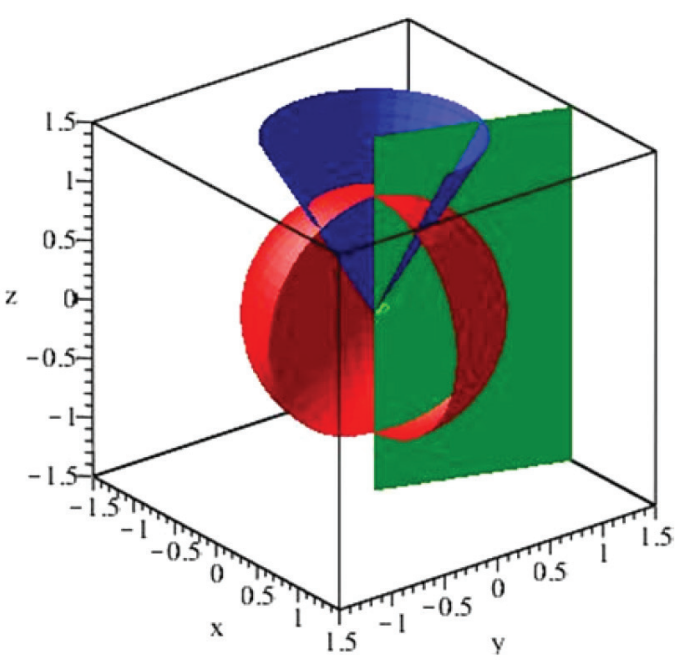

Figure 1. Definition of spherical polar coordinates: a sphere (red) of radius $r=1$ unit with its center at the origin cut open to show the interior; a circular cone (blue) of constant $\theta$ at angle $\pi / 6$ rad to axis $z$ and with its vertex at the origin, also cut open; a half-plane (green) of constant $\phi$ is at angle $3 \pi / 5 \mathrm{rad}$ to plane $x z$ for which $y=0$. The domains are $0 \leq r<\infty, 0 \leq \theta<\pi, 0 \leq \phi<2 \pi$

$$
\begin{aligned}
& \psi_{k, l, m}(r, \theta, \phi)=\sqrt{\frac{Z \pi \mu e^{2} k !}{\varepsilon_{0} h^{2}(k+2 l+1) !}}\left(\frac{2 \mu \pi e^{2} Z}{(k+l+1) h^{2} \varepsilon_{0}}\right)^{(l+1)} r^{l} \\
& \text { LaguerreL }\left(k, 2 l+1, \frac{2 \pi \mu e^{2} Z r}{h^{2} \varepsilon_{0}(k+l+1)}\right) e^{\left(-\frac{\pi \mu e^{2} Z r}{h^{2} \varepsilon_{0}(k+l+1)}\right)} e^{(i m \phi)} \\
& \sqrt{\frac{(2 l+1)(l-|m|) !}{(l+|m|) !}} \text { Legendre } P(l,|m|, \cos (\theta)) /(2(k+l+1) \sqrt{\pi})
\end{aligned}
$$

In this equation that is deliberately expressed in terms of fundamental physical constants appear as parameters quantum numbers radial $k$, as first argument of generalized Laguerre functions denoted LaguerreL with coordinate $r$, azimuthal $l$ as first argument of associated Legendre functions of the first kind and denoted LegendreP with coordinate $\theta$, and equatorial $m$ that is coefficient of equatorial angle $\phi$ and $i=\sqrt{ }-1$ in an exponential function. Other symbols are atomic number $Z$, reduced mass $\mu=m_{e} m_{p} /\left(m_{e}+m_{p}\right)$ of the atomic system for which $Z=1$ for $\mathrm{H}$ with a proton of mass $m_{\mathrm{p}}$ as atomic nucleus, electric charge $-e$ for an electron of mass $m_{\mathrm{e}}$, Planck constant $h$ and electric permittivity $\varepsilon_{0}$ of vacuum. In terms of quantum numbers specific for this coordinate system, energy quantum number $n$ from experiment is equal to $k+l+1$. These quantum numbers $k, l, m$ that arise directly in the solution of the Schroedinger equation in spherical polar coordinates are natural descriptors for this system because they specify the number of nodal surfaces associated with each respective coordinate $r, \theta, \phi$.

As that equation for an amplitude function as orbital comprises three independent variables as coordinates $r, \theta$, $\phi$, and one dependent variable as amplitude $\psi$, a plot of such a function would require four spatial dimensions, which is impracticable. We hence show a surface of constant $\psi$ at a value chosen to reveal the essential geometric features; the value of $\psi$ is explicitly chosen, here and below, such that the surface of $\psi^{2}$ at that value would contain about 0.99 of the total electronic charge density. For quantum numbers $k=0, l=1$, $m=0$, so $n=2$, the amplitude function (commonly known as orbital $2 p$ ) has this explicit algebraic form in terms of fundamental parameters:

$$
\psi_{0,1,0}(r, \theta, \phi)=\frac{\sqrt{2} e^{5}(\mu Z)^{\left(\frac{5}{2}\right)} \pi^{2} r e^{\left(-\frac{\pi \mu Z e^{2} r}{2 h^{2} \varepsilon_{0}}\right)} \cos (\theta)}{8 h^{5} \varepsilon_{0}{ }^{\left(\frac{5}{2}\right)}}
$$

A plot of the surface of this amplitude function or orbital, according to the stated criterion, appears in Figure 2. The surface comprises two separate hemispheres, of opposite phase on either side of plane $x y$ for which $z=0$, and with rounded edges; the overall nearly spherical shape is consistent with the fact that the Coulombic attraction between an electron and a proton has no angular dependence.

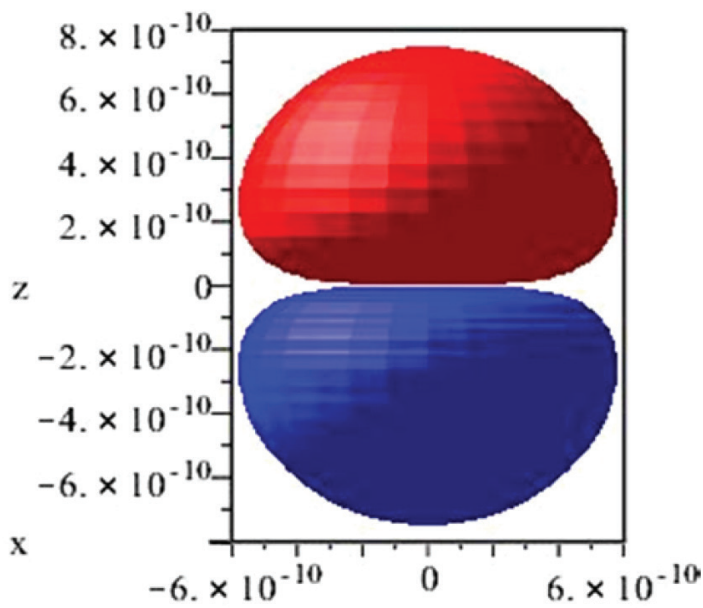

Figure 2. Surface of $\psi_{0,1,0}(r, \theta, \phi)= \pm 3.17 \times 10^{12} \mathrm{~m}^{-3 / 2}$; the upper lobe (red) has a positive phase, the lower lobe (blue) has a negative phase. The length scale on each axis is in terms of metre consistent with the SI units of the plotted function

\section{Paraboloidal Coordinates}

The spatial variables in this system ${ }^{8}$ are conventionally named $u, v$ and $\phi$ : a surface of constant $u$ corresponds to an infinite paraboloid, which is a parabola of revolution about axis $z$, opening downward and with its focus at the origin; a surface of constant $v$ corresponds to an infinite paraboloid about axis $z$ opening upward and with its focus also at the origin; a surface of constant $\phi$ again corresponds to an infinite half-plane from axis $z$. Figure 3 illustrates these features.

Solutions $\psi_{n_{1}, n_{2}, m}(u, v, \phi)$ to the Schroedinger equation independent of time in these paraboloidal coordinates, i.e. the orbitals, are expressible in terms of fundamental physical constants as

$$
\begin{aligned}
& \Psi_{n_{1}, n_{2}, m}(u, v, \phi)=(-1)^{|m|} \sqrt{\frac{Z \pi \mu e^{2}}{\varepsilon_{0} h^{2}}} \sqrt{\frac{2 n_{1} ! n_{2} !}{\left(n_{1}+|m|\right) !\left(n_{2}+|m|\right) !}} \\
& \left(\frac{\pi Z e^{2} \mu}{h^{2} \varepsilon_{0}\left(|m|+n_{1}+n_{2+1}\right)}\right)^{(1+|m|)}(u v)^{|m|} e^{\left(-\frac{\pi Z e^{2} \mu\left(u^{2}+v^{2}\right)}{2 h^{2} \varepsilon_{0}\left(|m|+n_{1}+n_{2}+1\right)}\right) e^{(i m \phi)}} \\
& \text { LaguerreL }\left(n_{1},|m|, \frac{\pi Z e^{2} \mu u^{2}}{h^{2} \varepsilon_{0}\left(|m|+n_{1}+n_{2}+1\right)}\right) \\
& \text { LaguerreL }\left(n_{2},|m|, \frac{\pi Z e^{2} \mu v^{2}}{h^{2} \varepsilon_{0}\left(|m|+n_{1}+n_{2}+1\right)}\right) /\left(\sqrt{2 \pi}\left(|m|+n_{1}+n_{2}+1\right)\right)
\end{aligned}
$$

In this equation appear quantum numbers $n_{1}$, as first argument of a generalized Laguerre function denoted LaguerreL of which the spatial variable is $u, n_{2}$ as first argument of a generalized Laguerre function of which the spatial variable is $v$, and equatorial 


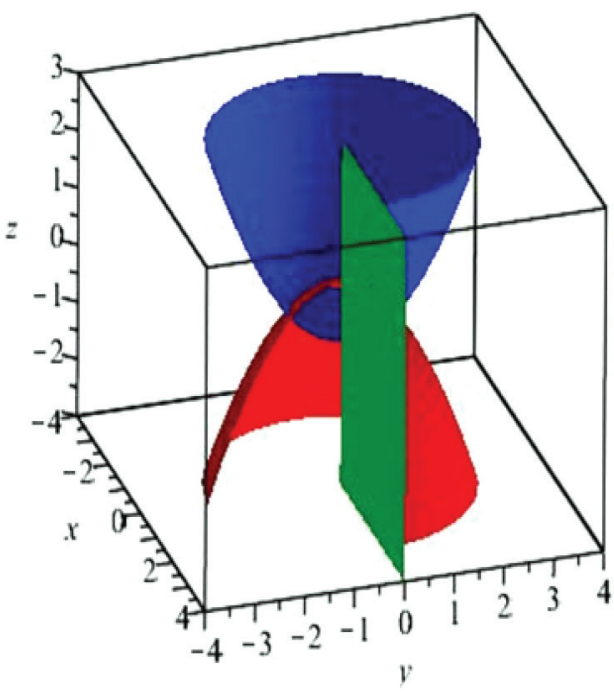

Figure 3. Definition of paraboloidal coordinates: a paraboloid (red) for $u=1$ unit with its focus at the origin and opening downward, cut open to show the interior; a paraboloid (blue) for $v=1$ unit with its focus at the origin and opening upward, likewise cut open; a half-plane (green) for $\phi=0$ is, accordingly, in plane $x z$ for which $y=0$. The domains are $0 \leq u, v<\infty, 0 \leq \phi<2 \pi$

$m$ that is again coefficient of equatorial angle $\phi$ and $\sqrt{ }-1$ in an exponential function. Experimental energy quantum number $n=n_{1}+n_{2}+|m|+1$, in terms of quantum numbers specific for this coordinate system. With the same criterion as for $\psi_{0,1,0}(r, \theta, \phi)$ in Figure 2, we show a surface of constant $\psi_{0,1,0}(u, v, \phi)$ at a value chosen to reveal the essential geometric features. For quantum numbers $n_{1}=0, n_{2}=1, m=0$, so that energy quantum number $n=2$, the amplitude function has this explicit algebraic form in terms of fundamental parameters:

$\psi_{0,1,0}(u, v, \phi)=\frac{Z^{3} \mu^{3} e^{6} \pi^{2} e^{\left(-\frac{Z e^{2} \pi \mu\left(u^{2}+v^{2}\right)}{2 h^{2} \varepsilon_{0}}\right)}\left(\pi Z e^{2} \mu v^{2}-2 h^{2} \varepsilon_{0}\right)^{2}}{64 h^{10} \varepsilon_{0}^{5}}$

A plot of the surface of this amplitude function. which is an orbital, according to the stated criterion, appears in Figure 4. The surface comprises two lobes, the upper one small, nearly ellipsoidal and of negative phase about axis $z$, the lower one large and of positive phase.

\section{Ellipsoidal Coordinates}

The coordinates in this system, ${ }^{9}$ also called prolate spheroidal, are conventionally named $\xi, \eta, \phi$ : a surface of $\xi=2$ corresponds to a finite ellipsoid, which is an ellipse of revolution about axis $z$, with one focus at the origin and a second focus at $z=2$; for $\xi=1$ such an ellipsoid degenerates into a line along axis $z$ from $z=0$ to $z=2$; for $\xi=a>1$, the ellipsoid has axis $z$ as its major axis and extends from $-a$ to $a+2$ along that axis. A surface of constant $\eta$ is an infinite hyperboloid coincident with axis $-z$ for $\eta=-1$ and its vertex at the origin, coincident with axis $+z$ for $\eta=+1$ and with its vertex at $z=2$, and degenerating into a plane at $z=1$ for $\eta=0$; a surface of constant $\phi$ again corresponds to an infinite half-plane from axis $z$. Figure 5 illustrates these features.

The solutions $\psi_{n, \lambda, m}(\xi, \eta, \phi)$ of the Schroedinger equation independent of time in these ellipsoidal coordinates, i.e. the orbitals, are expressible as

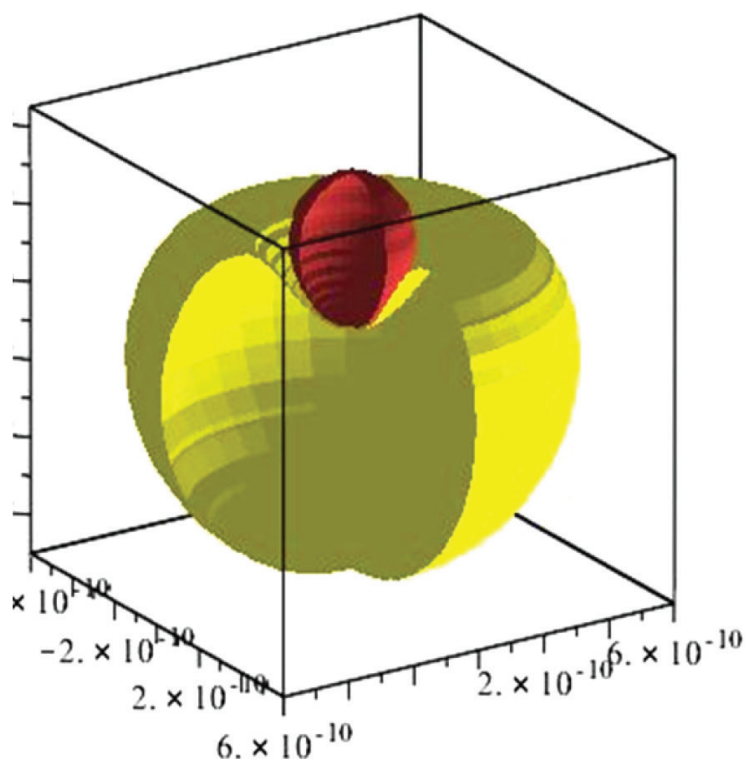

Figure 4. Surface of $\psi_{0,1,0}(u, v, \phi)= \pm 1.46 \times 10^{13} \mathrm{~m}^{-3 / 2}$; the lower lobe (yellow) has a positive phase, the upper lobe (brown) negative phase; both are cut open to reveal the details of the surfaces. The length scale on each axis is in terms of metres consistent with SI units of the plotted function

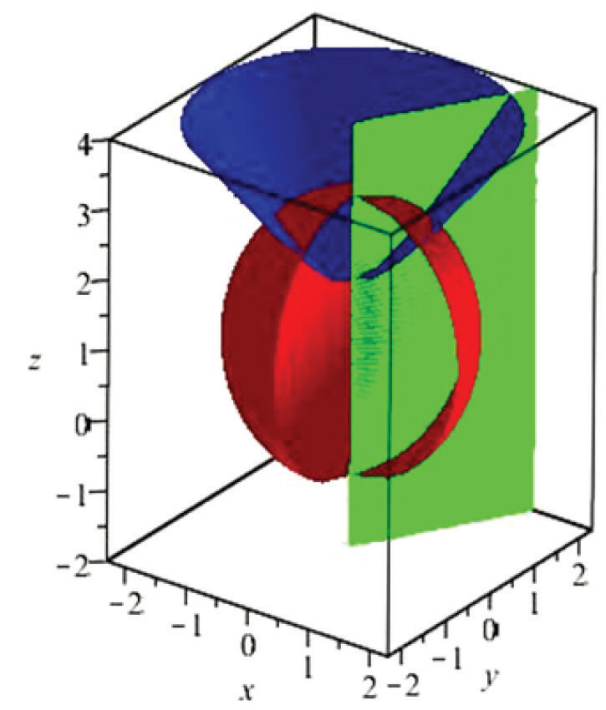

Figure 5. Definition of ellipsoidal coordinates: surfaces of an ellipsoid (red) with $\xi=2$, an hyperboloid (blue) with $\eta=\pi / 4$ rad, both cut open to reveal the inner details, and a half-plane (green) with $\phi=\pi / 3$ rad. The axes are scaled in unit $a_{\mu}$, which signifies a Bohr radius corrected for reduced mass $\mu$ of the system. The domains are $1 \leq \xi<\infty,-1 \leq \eta \leq 1,0 \leq \phi<2 \pi$

$$
\begin{gathered}
\psi_{n, \lambda, m}(\xi, \eta, \phi)=N\left(\left(\xi^{2}-1\right)\left(\eta^{2}-1\right)\right)^{\left(\frac{|m|}{2}\right)_{e}\left(-\frac{d Z(\xi+\eta+1)}{2 a_{\mu} n}\right)_{e}(i m \phi)} \\
\operatorname{HeunC}\left(-\frac{2 d Z}{a_{\mu} n},|m|,|m|, \frac{2 Z d}{a_{\mu}}, \frac{m^{2}}{2}-\frac{Z d}{a_{\mu}}-\lambda(\lambda+1)+f_{n, \lambda, m}(d)-\frac{Z^{2} d^{2} n^{2}}{4 a_{\mu}^{2}}, \frac{\xi}{2}+\frac{1}{2}\right) \\
\operatorname{HeunC}\left(-\frac{2 d Z}{a_{\mu} n},|m|,|m|, \frac{2 Z d}{a_{\mu}}, \frac{m^{2}}{2}-\frac{Z d}{a_{\mu}}-\lambda(\lambda+1)+f_{n, \lambda, m}(d)-\frac{Z^{2} d^{2}}{4 a_{\mu}^{2} n^{2}}, \frac{\eta}{2}+\frac{1}{2}\right) / \sqrt{2 \pi}
\end{gathered}
$$

In this equation that is expressed in terms of $a_{\mu}$, which signifies a Bohr radius $a_{0}$ corrected for the reduced mass of the system, $a_{\mu}=\varepsilon_{0} h^{2} /\left(\pi \mu Z e^{2}\right)$ with $a_{\mu}=m_{e} a_{0} / \mu$ for effective reduced mass $\mu$ of the atomic system, appear normalizing factor $N$ and quantum numbers $n, \lambda$ and equatorial $m$ that is again coefficient of equatorial angle $\phi$ and 
$i=\sqrt{ }-1$ in an exponential function; the distance between the two foci of the ellipsoid is given symbol $d$, and all amplitude functions depend sensitively on the value of this parameter. Because Schroedinger's equation in these coordinates is a special case of Heun's differential equation, confluent functions HeunC naturally appear in the solutions; there is no singularity of these functions within the defined domains in the caption of Figure 5. In this formula energy quantum number $n$ appears directly, but, in an alternative and partial solution in terms of variables $\xi$ and $\eta$ in separate series, ${ }^{10} n=n_{\xi}+n_{\eta}+|m|+1$.

For quantum numbers $n=2, \lambda=0, m=0$, the amplitude function or orbital has this explicit algebraic form in terms of Bohr radius $a_{\mu}$ :

$$
\begin{gathered}
\psi_{2,0,0}(\xi, \lambda, m)=N e^{\left(-\frac{d Z(\xi+\eta+1)}{4 a_{\mu}}\right)} \\
\operatorname{HeunC}\left(-\frac{Z d}{a_{\mu}}, 0,0, \frac{2 Z d}{a_{\mu}},-1-\frac{Z d}{a_{\mu}}+\frac{d^{2}}{16 a_{\mu}}+\frac{\sqrt{\frac{d^{2}}{a_{\mu}{ }^{2}}+4}}{2}-\frac{Z^{2} d^{2}}{16 a_{\mu}{ }^{2}}, \frac{\xi}{2}+\frac{1}{2}\right) \\
\operatorname{HeunC}\left(-\frac{Z d}{a_{\mu}}, 0,0, \frac{2 Z d}{a_{\mu}},-1-\frac{Z d}{a_{\mu}}+\frac{d^{2}}{16 a_{\mu}}+\frac{\sqrt{\frac{d^{2}}{a_{\mu}{ }^{2}}+4}}{2}-\frac{Z^{2} d^{2}}{16 a_{\mu}{ }^{2}}, \frac{\eta}{2}+\frac{1}{2}\right) / \sqrt{2 \pi}
\end{gathered}
$$

According to the same criterion as for $\psi_{0,1,0}(r, \theta, \phi)$ in Figure 2, we show in Figure 6 a surface of this amplitude function $\psi_{2,0,0}(\xi, \eta, \phi)$, at a value of $d$ chosen to reveal the essential geometric features. The surface comprises two lobes, a small lobe nearly ellipsoidal and of negative phase along axis $z$, and a large lobe of positive phase. At distances $d<a_{\mu} / 10$, the inner lobe begins as a sphere with its center at the origin (not shown) inside a concentric shell of amplitude of opposite phase; with increasing $d$ the lobe assumes an ellipsoidal shape and moves upward along positive axis $z$ and eventually separates from the large lobe as in Figure 4.

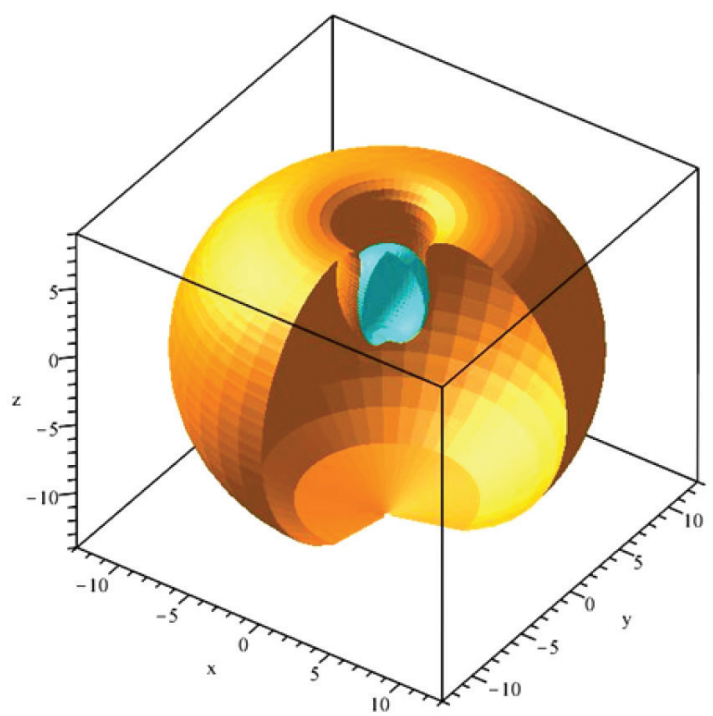

Figure 6. Surface of $\psi_{2,0,0}(\xi, \eta, \phi)= \pm 2.13 \times 10^{-4} a_{\mu}{ }^{-3 / 2}$ at $d=5 a_{\mu}$; the small lobe (light blue) of nearly ellipsoidal shape has a negative phase, the large lobe (coral) has a positive phase; both are cut open to reveal the inner details. The axes are scaled in unit $a_{\mu}$

\section{Spheroconical Coordinates}

The coordinates in this system, ${ }^{11}$ also called spheroconal, are conventionally named $\xi, r, \eta$ : a surface of constant $r$ corresponds to a finite sphere of that radius, as in spherical polar coordinates; a surface of constant $\xi$ corresponds to an infinite double cone with its apices at the origin and oriented about axis $z$, whereas a surface of constant $\eta$ corresponds to an infinite double cone with its apices at the origin and oriented about axis $x$. Each double cone has an elliptical cross section in any plane perpendicular to its axis. These features are illustrated in Figure 7.

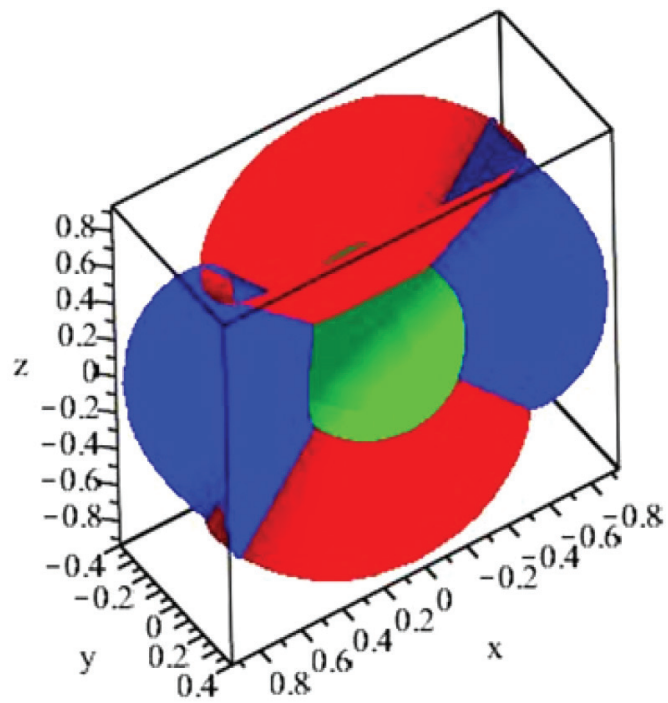

Figure 7. Definition of spheroconical coordinates: surfaces of a sphere (green) centered at the origin with $r=2 / 5$ units, an infinite double cone (red) of elliptical cross section about axis $z$ with $\xi=1 / 4$, and a second infinite double cone (blue) of elliptical cross section about axis $x$ with $\eta=1 / 4$. The domains are $0 \leq r<\infty,-1 / \sqrt{ } 2 \leq \xi, \eta \leq+1 / \sqrt{ } 2$

The solutions $\psi_{k, l, \mathrm{k}}(\xi, r, \eta)$ to the Schroedinger equation independent of time in these spheroconical coordinates, i.e. the orbitals, are expressible as

$$
\begin{gathered}
\psi_{k, l, \mathrm{\kappa}}(\xi, r, \eta)=N \sqrt{\frac{Z k !(2+2 l+k)}{a_{\mu}(2+2 l+k) !}}\left(\frac{2 Z}{a_{\mu}(k+l+1)}\right)^{(l+1)} r^{l} e^{\left(-\frac{Z r}{a_{\mu}(k+l+1)}\right)} \\
\text { LaguerreL }\left(k, 2 l+1, \frac{2 Z r}{a_{\mu}(k+l+1)}\right) \operatorname{HeunG}\left(-1, \kappa,-\frac{l}{2}, \frac{1}{2}+\frac{l}{2}, \frac{1}{2}, \frac{1}{2},-2 \xi^{2}\right) \\
\operatorname{HeunG}\left(-1,-\kappa,-\frac{l}{2}, \frac{1}{2}+\frac{l}{2}, \frac{1}{2}, \frac{1}{2},-2 \eta^{2}\right) /(k+l+1)
\end{gathered}
$$

In this formula appear normalizing factor $N$ and three quantum numbers, $k$ and $l$ that appear in associated Laguerre functions as for spherical polar coordinates, and $\kappa$ that appears only in the two general Heun functions with $l$; the latter functions arise because the ordinary-differential equations for $\xi$ and $\eta$, after separation of the variables in Schroedinger's partial-differential equation in this system of coordinates, are instances of Heun's differential equation. Energy quantum number $n=k+l+1$, in terms of quantum numbers specific for this coordinate system, so is independent of third quantum number $\kappa$. The most notable property of these amplitude functions in spheroconical coordinates is that, with $N$ real, these orbitals have no complex character: there is no factor $\mathrm{e}^{\mathrm{i} m \dot{ }}$ in any amplitude function that might bestow some imaginary component, unlike the functions in coordinates of the preceding three sets. This property warrants an expanded utilization of these functions, but calculations with conventional quantum-chemical software involve no imaginary quantities in any case.

For quantum numbers $n=1, l=0, \kappa=1$, the amplitude function 
has this explicit algebraic form in terms of Bohr radius $a_{\mu}$, as above, with an explicit numerically derived normalizing factor.

$$
\begin{aligned}
\psi(0,0,1)= & 5181464 e^{\left(-\frac{r}{a_{\mu}}\right)} \text { LaguerreL }\left(0,1, \frac{2 r}{a_{\mu}}\right) \operatorname{HeunG}\left(-1,1,0, \frac{1}{2}, \frac{1}{2}, \frac{1}{2},-2 \xi^{2}\right) \\
& \operatorname{HeunG}\left(-1,-1,0, \frac{1}{2}, \frac{1}{2}, \frac{1}{2},-2 \eta^{2}\right) /\left(11269745 a_{\mu}\left(\frac{3}{2}\right)\right)
\end{aligned}
$$

With the same criterion as for $\psi_{0,1,0}(r, \theta, \phi)$ in Figure 2, Figure 8 shows a surface of this amplitude function $\psi_{0,0,1}(\xi, r, \eta)$, according to the stated common criterion, to reveal the essential geometric features of this orbital. The surface comprises two lobes, one a closed double conical lobe about axis $x$ on either side of the origin with both apices at the origin, and the other a large torus of elliptical shape about that axis $x$ and hence surrounding the cones near the origin.

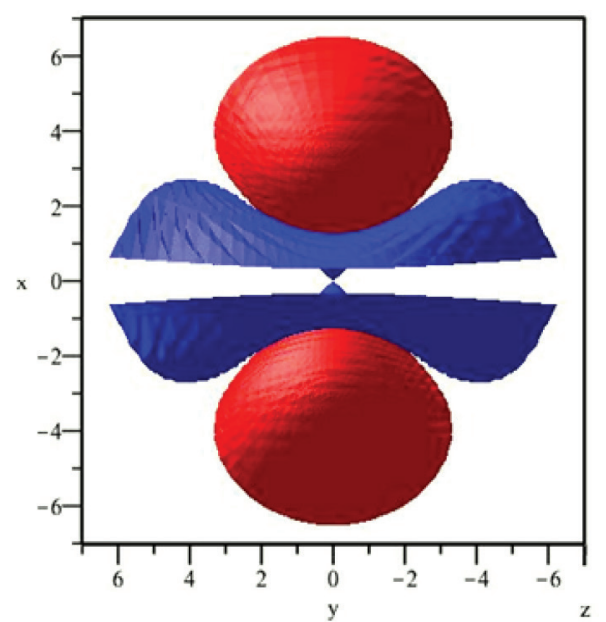

Figure 8. Surface of $\psi_{0,0,1}(\xi, r, \eta)= \pm 0.0041 a_{\mu}{ }^{-3 / 2}$; the double conical lobe (red) with its apices at the origin has a positive phase; the torus (blue) about axis $x$ has a negative phase. The axes are scaled in unit $a_{\mu}$. The surface is cut open to show the interior

\section{Summary of coordinate systems}

The impact of these eight figures on a reader should be that solutions to the Schroedinger equation for the hydrogen atom, and hence orbitals by definition, exist for coordinates in four distinct and independent sets. Each respective set of corresponding orbitals has its intrinsic three quantum numbers, not all common; surfaces of constant amplitude $\psi$ have disparate shapes. There is no one and unique set of orbitals for the hydrogen atom, and so there is no one and unique set of their quantum numbers. Many further pictures of surfaces of amplitude functions in the four systems of coordinates are available elsewhere, with other properties and applications of these functions. ${ }^{7-9,11}$

Schroedinger's equation independent of time is solvable also in cartesian coordinates $x, y, z$ with also $r$ as $\sqrt{x^{2}+y^{2}+z^{2}},{ }^{12}$ but only a partial separation of spatial variables is practicable; because plots of these amplitude functions, with the same quantum numbers $k, l, m$ as in spherical polar coordinates, exhibit the same shapes and sizes of surfaces as the corresponding surfaces based on spherical polar coordinates, these Cartesian amplitude functions must be considered to be merely a variant of the functions in spherical polar coordinates.

For the system of ellipsoidal coordinates, amplitude functions $\psi_{n, \lambda, m}(\xi, \eta, \phi)$ exhibit limiting values that depend on distance $d$ between the foci of the ellipsoid: as $d \rightarrow 0$, they become equivalent to amplitude functions $\psi_{k, l, m}(r, \theta, \phi)$ in spherical polar coordinates or linear combinations of these functions; as $d \rightarrow \infty$, they become equivalent to amplitude functions $\psi_{n_{1}, n_{2}, m}(u, v, \phi)$ in paraboloidal coordinates or their linear combinations, but at finite intermediate distance $d$ these amplitude functions have characteristic shapes distinct from those of the limiting cases. One can readily recognize the similarity between Figure 6 as an intermediate case in relation to Figure 4 as a limiting case for these amplitude functions in ellipsoidal coordinates, such that $\psi_{0,1,0}(\xi, \eta, \phi) \rightarrow \psi_{0,1,0}(u, v, \phi)$ as $d \rightarrow \infty$; the other limiting case in this instance is $\psi_{1,0,0}(r, \theta, \phi)$ as $d \rightarrow 0$. For the ground state, $1{ }^{2} \mathrm{~S}_{1 / 2}$, of the hydrogen atom, the corresponding surfaces of all respective amplitude functions in the four systems are perfect spheres.

The amplitude functions in spherical polar and paraboloidal coordinates are presented above in terms of the fundamental physical constants and atomic parameters so as to provide, for a reader, a formulation of these functions alternative to what other reference sources typically present. ${ }^{13}$ For coordinates in the other two systems, the formulae become expressed more compactly with the use of Bohr radius $a_{\mu}$ corrected for reduced mass $\mu$, but in all cases the formulae are exact, within a context of the Schroedinger equation, and are exactly applicable to an atom of atomic number $Z$ with only one electron, and the corresponding value of reduced mass $\mu$ for that atomic system. In no case is the quantum-mechanical nature of the atomic nucleus or its finite volume taken into account; the motion of the center of mass of the atomic system is assumed to be separated and treated appropriately. Apart from the latter qualm, the preceding information is precisely suitable for inclusion in a textbook of physical chemistry of which the authors might reasonably seek to present an unbiased treatment of the hydrogen atom according to wave mechanics, but is obviously unsuitable for introductory chemistry. Any responsible author of a textbook of general chemistry should nevertheless be aware of, and knowledgeable about, these fundamental aspects, namely the multiplicity of sets of orbitals, before embarking on a discussion involving orbitals in any manner, shape or form.

The derivation of the orbitals in all cases is based on a Coulombic attraction between a single electron, bearing a single negative electric charge, and an atomic nucleus, positively charged, which might possess multiple protons according to atomic number $Z$, such as for $\mathrm{He}^{+}, \mathrm{Li}^{2+}, \ldots$ Factor $Z$ in all general equations for amplitude functions in terms of coordinates in the four primary systems takes into account the fact that these functions are legitimately applicable to any isolated atomic system involving one electron within the bound of a non-relativistic frame. The use of any such function for an atomic system involving more than one electron signifies an ignoring of the Coulombic repulsion between electrons at the same time that these electrons are subject to a Coulombic attraction to an atomic nucleus, in violation of the fundamental laws of physics. We reiterate that the use of orbitals as basis functions in proper quantitative quantumchemical calculations is in no way contrary to this principle, because the repulsive effects are taken into account separately in a manner that is beyond the scope of qualitative arguments here or in introductory chemistry.

In a discussion of the electronic structure of an atom with more than one electron, one should logically apply an amplitude function derived for that particular system. For instance, for the hydride ion or the helium atom, either of which has two electrons associated with a single atomic nucleus, an exact amplitude function, implicitly in atomic units, for the ground electronic state near a point of coalescence is expressed as follows in terms of distances $r_{1}$ and $r_{2}$ between one or other electron and that nucleus and distance $r_{12}$ between the two electrons. ${ }^{14}$ 


$$
\begin{gathered}
\psi=1-Z\left(r_{1}+r_{2}\right)+\frac{1}{2} r_{12}+\frac{1}{12}\left(4 Z^{2}-2 E+1\right) r_{12}^{2}+Z^{2} r_{1} r_{2} \\
+\frac{1}{6} Z\left(r_{1}^{2}+r_{2}{ }^{2}-r_{12}{ }^{2}\right) \ln \left(\frac{r_{1}+r_{2}-r_{12}}{r_{1}+r_{2}+r_{12}}\right)-\frac{1}{3} Z r_{12}\left(r_{1}+r_{2}\right) \\
+\frac{2}{3}\left(r_{1}^{2}+r_{2}^{2}\right) \cos (\alpha) \arctan h\left(\frac{r_{12} \sqrt{1+\sin (\alpha)}}{\sqrt{r_{1}^{2}+r_{2}^{2}} \cos (\alpha)}\right)-\frac{2}{3} r_{12} \sqrt{r_{1}^{2}+r_{2}^{2}} \sqrt{1+\sin (\alpha)} \\
-\frac{2}{3}\left(r_{1}^{2}+r_{2}^{2}\right) \cos (\alpha) \arctan h\left(\frac{r_{12}\left(2 r_{1}^{2}+2 r_{2}^{2}-r_{12}{ }^{2}\right)}{\left(r_{1}^{2}+r_{2}{ }^{2}\right) \cos (\alpha)}\right)+\frac{2}{3} r_{12} \sqrt{2 r_{1}^{2}+2 r_{2}^{2}-r_{12}^{2}}
\end{gathered}
$$

Here atomic number $\mathrm{Z}$ is 2 for $\mathrm{He}$ and 1 for $\mathrm{H}^{-} ; E$ represents the energy of the ground state of the system; angle $\alpha$ denotes the value of $\arcsin \left(2 r_{1} r_{2} /\left(r_{1}^{2}+r_{2}^{2}\right)\right)$ subtended at the nucleus. For a carbon atom there would be six distances $r_{j}$ from the nucleus and fifteen distances $r_{j k}$ between electrons; the resulting algebraic expression of an exact amplitude function would have intolerable length. Although the problem of many bodies interacting in a quantum-mechanical system is solvable in principle,,$^{15}$ the result for even the He atom, above, is intractable for any practical purpose; an effort to produce a result for an atom with many electrons would be futile. These circumstances in no way excuse, however, a qualitative or pictorial use of amplitude functions derived for the hydrogen atom, i.e. orbitals, in an application to an atomic system comprising multiple electrons, which would amount to extrapolation from a point.

\section{Schemes of electronic structure other than orbitals}

If one takes into account that the distribution of the density of electronic charge within a molecule or within a unit cell of a crystal is continuous and varies gradually in space in the vicinity of atomic nuclei, any classification of electrons becomes meaningless. Not only is there no $1 s, 2 p, \ldots$ electron, there is no valence electron, no lone pair and so forth, because electrons are fundamentally indistinguishable. ${ }^{5}$

Both experimental measurement and accurate calculation that reproduces that measurement, either of which is subject to error, indicate that midway between the two protons in molecular ${ }^{1} \mathrm{H}_{2}$, at its equilibrium internuclear distance, the density of electronic charge is slightly - less than 10 per cent, but significantly - greater than would be the hypothetical case of two non-interacting ${ }^{1} \mathrm{H}$ atoms at the same interprotonic distance. ${ }^{16}$ Although that increased electronic charge between the two protons occurs at an expense of a slight depletion of electronic charge on the other side of each proton, the attribution of a chemical bond to a pair of electrons between the two atomic nuclei is clearly a gross exaggeration. The dihydrogen molecule, stable with respect to dissociation into two hydrogen atoms by about $4.48 \mathrm{eV}$, is an experimental fact; that dissociation energy is less than one third the ionization energy of a hydrogen atom. For the dihydrogen molecular cation, ${ }^{1} \mathrm{H}_{2}{ }^{+},{ }^{17}$ the dissociation energy is about $2.65 \mathrm{eV}$, slightly more than half the value for the neutral molecule. The dihelium molecule, ${ }^{4} \mathrm{He}_{2}$, is only weakly bound, but it exists and has a bound vibrational state. In contrast the dihelium cation, ${ }^{4} \mathrm{He}_{2}{ }^{+}$, is strongly bound: its dissociation energy, about $3.1 \mathrm{eV}$, is similar to that of ${ }^{1} \mathrm{H}_{2}{ }^{+}$.

For the next homonuclear dinuclear molecule in order of increasing atomic number, dilithium ${ }^{7} \mathrm{Li}_{2}$, one might be tempted to insinuate a typical chemical bond involving two electrons, notwithstanding the caveat above; this species is stable to dissociation by $1.04 \mathrm{eV}$, whereas, for the dinuclear molecular cation ${ }^{7} \mathrm{Li}_{2}{ }^{+}$for which only a one-electron chemical bond would seem to be possible, the dissociation energy is much greater, $1.4 \mathrm{eV} .{ }^{18}$ For the corresponding molecular anion, ${ }^{7} \mathrm{Li}_{2}{ }^{-}$, the calculated dissociation energy is $0.86 \mathrm{eV}$, only slightly less than for the neutral molecule. If these ideas about an electron-pair bond fail in the simplest cases, quite apart from the complications such as for the boron hydrides, how can one have confidence that their more general application is worth the effort to rationalize these effects?

The idea of an octet of valence electrons that Abegg first reported ${ }^{19}$ was subsequently elaborated by Kossel ${ }^{20}$ and by Lewis. ${ }^{21}$ With the additional concept of electron spin dictated by the Pauli principle, that octet became viewed as four pairs of electrons, one electron of each spin within each pair. An alternative approach involved a double quartet of valence electrons, electrons in each quartet having the same spin and opposite the spin of electrons in the other quartet. ${ }^{22}$ In all three cases the indistinguishability of electrons, in differentiating valence electrons from other electrons, is contradicted.

The extent of an orbital is infinite, even though there might exist surfaces of zero amplitude between regions of positive and negative phase, such as is discernible in Figure 2 for instance. One attempt to define a localized region of space in which an electron pair was likely to be found was implemented in a loge theory, so to partition the space surrounding atomic nuclei within a molecule into various loges. ${ }^{23}$ This endeavor seemed successful in the case of $\mathrm{LiH}$ but was increasingly unsatisfactory for $\mathrm{BeH}_{2}, \mathrm{BH}_{3}$ and $\mathrm{CH}_{4}$; as those volumes designated as loges were in any case evaluated as a result of extensive quantum-chemical calculations, their applicability to explain molecular structure in general chemistry is minimal. Another region of space in the valence shell in which an electron pair is most probably to be found has been called an electron-pair domain; ${ }^{24}$ such a domain is based on an assumed electronic structure, and represents yet another circular argument. Any such partition of space surrounding atomic nuclei is based on arbitrary criteria, not subject to direct experimental confirmation.

\section{Relation between molecular structure and quantum mechanics}

To explain the properties and reactions of chemical substances, the structure at an atomic level of molecules and materials is the most important aspect of contemporary chemistry. Previous authors discussing the use of orbitals (Part I) seemed to ignore or to dismiss the fact that molecular structure is incompatible with quantum mechanics. ${ }^{25}$ A thorough calculation on a molecule in which all particles - both electrons and atomic nuclei - are treated equitably, i.e. according to rigorous quantum mechanics, yields no structure. ${ }^{26}$ The application of orbitals, as algebraic formulae that are artefacts of one particular method of quantum mechanics, to explain molecular structure has thus no logical foundation. ${ }^{27}$ This argument is more than semantic: one cannot state that a molecule has a particular shape because of orbitals; one can also not state that calculated stationarystate amplitude functions can be discussed in terms of shape. ${ }^{28} \mathrm{~A}$ molecular structure might be best thought of as a useful metaphor, ${ }^{29}$ but the chemical and physical reality is the structure of molecules and materials, for instance, from x-ray crystallography, not their purported orbitals.

Another conundrum is that most practicing quantum chemists continue to undertake structural calculations without acknowledging this principle of incompatibility; ${ }^{30}$ in contrast, philosophers of chemistry readily accept it. ${ }^{31}$ Whereas some educators agree that it should be discussed with students, ${ }^{28}$ for the most part it has been ignored in chemical education in the twenty-first century.

\section{CONCLUSIONS}

We end as we begin, by recalling Dirac's perceptive dictum, "science is concerned only with observable things". ${ }^{1}$ Orbitals are not observable $;, 32$ hence leave these algebraic formulae to the physicists, if they want. ${ }^{33}$ In a chemical context, electrons might be tangible 
things, but individual electrons, or even purported electron pairs, are not observable - only the total density of electronic charge in the vicinity of atomic nuclei. What are observable, and on which the entire edifice of chemistry is based, are the atomic centers in a molecule or material, each comprising an atomic nucleus of positive electric charge embedded within its associated environment of continuous negative electronic charge density.

A reader should bear in mind that, for the teaching of chemistry with orbitals, proponents and opponents alike have been under an illusion that we might call the conundrum of chemistry and quantum mechanics; contrary to that illusion, molecular structure, which is the basis of understanding all modern chemistry, is incompatible with quantum mechanics..$^{29}$ Any use of quantum-mechanical arguments, and their artefacts such as orbitals, to explain molecular structure is hence profoundly illogical. ${ }^{27}$ Yes, quantum-mechanical methods, with a classical treatment of relative nuclear positions - so semiempirically, are useful to refine molecular structures, but so can methods of molecular mechanics with no quantum-mechanical provenance whatsoever. ${ }^{34}$

In Part I, we present mostly opinions in a continuing debate about the teaching of orbitals in general chemistry, whereas Part II presents mathematical evidence that the textbooks ignore. In Part III, we provide guidelines to encompass the pedagogical discussion in Part I and to combine it with the mathematical realities in Part II.

\section{ACKNOWLEDGEMENTS}

El Centro de Investigaciones en Productos Naturales (CIPRONA) and la Escuela de Química, Universidad de Costa Rica (UCR), provided support. We thank several students and professors at UCR for helpful discussion.

\section{REFERENCES}

1. Dirac, P. A. M.; The Principles of Quantum Mechanics, Oxford University Press: London, UK, 1967, 3.

2. Brown, T. L.; LeMay, H. E.; Brusten, B. E.; Murphy, C. J.; Woodward, P. M.; Chemistry: The Central Science, Pearson: Boston, USA, 2012, 2.

3. Kalnins, E. G.; Miller, W.; Winternitz, P.; SIAM J. Appl. Math. 1976, 30, 630 .

4. Schrödinger, E. R. J. A.; Quantisation as a Problem of Proper Values, Parts I-IV, Annalen der Physik, 1926; Collected Papers on Wave Mechanics, AMS Chelsea: Providence, RI, USA, 2010, p. 102.

5. Truhlar, D. G.; Hiberty, P. C.; Shaik, S.; Gordon, M. S.; Danovich, D.; Angew. Chem. Int. Ed. 2019, 58, 12332.

6. McKelvey, D. R.; J. Chem. Educ. 1983, 60, 112.

7. Ogilvie, J. F.; Revista de Ciencia y Tecnologia 2016, 32, 1, available at http://revistas.ucr.ac.cr/index.php/cienciaytecnologia, accessed in October 2020.
8. Ogilvie, J. F.; Revista de Ciencia y Tecnologia 2016, 32, 25, available at http://revistas.ucr.ac.cr/index.php/cienciaytecnologia, accessed in October 2020.

9. Ogilvie, J. F.; Revista de Ciencia y Tecnologia 2016, 32, 40, available at http://revistas.ucr.ac.cr/index.php/cienciaytecnologia, accessed in October 2020.

10. Kereselidze, T.; Machaviani, Z. S.; Chkadua, G.; Eur. Phys. J. D. 2011, 63,81 .

11. Ogilvie, J. F.; Revista de Ciencia y Tecnologia 2016, 32, 54, available at http://revistas.ucr.ac.cr/index.php/cienciaytecnologia, accessed in October 2020.

12. Ogilvie, J. F.; Revista de Ciencia y Tecnologia 2019, 35, 8, available at http://revistas.ucr.ac.cr/index.php/cienciaytecnologia, accessed in October 2020

13. Cook, D. B.; Fowler, P. W.; Am. J. Phys. 1981, 49, 857.

14. Witek, H.; Lecture, Universidad de Costa Rica, Escuela de Química, 2014, Sept. 11.

15. Knirk, D. L.; Phys. Rev. Lett. 1974, 32, 651.

16. Moszynski, R.; Krzysztof, S.; J. Phys. B: At. Mol. Phys. 1987, 20, 4347.

17. Rioux, F.; Chem. Educ. 1997, $2,1$.

18. Linnett, J. W.; Lecture, University Chemical Society, University of Cambridge, 1965, Oct. 6.

19. Abegg, R.; Z. Anorg. Chem. 1904, 39, 330.

20. Kossel, W.; Ann. Phys. 1916, 49, 229.

21. Lewis, G. N.; J. Amer. Chem. Soc. 1916, 38, 762.

22. Linnett, J. W.; The Electronic Structure of Molecules - a New Approach, Methuen: London, 1964, 22.

23. Aslangul, C.; Constanciel, R.; Daudel, R.; Kottis, P.; Adv. Quant. Chem. 1972, 6, 93.

24. Gillespie, R. J.; Spencer, J. N.; Moog, R. S.; J. Chem. Educ. 1996, 73, 622.

25. Woolley, R. G.; Adv. Phys. 1976, 25, 27.

26. Cafiero, M.; Adamowicz, L.; Chem. Phys. Lett. 2004, 387, 136.

27. Primas, H.; Quantum Chemistry and Reductionism: Lecture Notes in Chemistry, Vol. 24, Springer-Verlag: Berlin, Germany, 1981.

28. Weininger, S. J.; J. Chem. Educ. 1984, 61, 939.

29. Woolley, R. G.; J. Chem. Educ. 1985, 62, 1082.

30. Sutcliffe, B. T.; Cryst. Rev. 2006, 12, 335.

31. Sutcliffe, B. T.; Woolley, R. G.; Atoms and Molecules in Classical Chemistry and Quantum Mechanics, Elsevier: Amsterdam, Netherlands, 2012, pp. 387-426.

32. Ogilvie, J. F.; J. Chem. Educ. 1990, 67, 280.

33. Omelianovsky, M. E.; Found. Phys. 1972, 2, 223.

34. Lewars, E. G.; Computational Chemistry: Introduction to the Theory and Applications of Molecular and Quantum Mechanics, Springer International Publishing: Switzerland, 2016, p. 121. 